\title{
Behavioral palatability of dietary fatty acids correlates with the intracellular calcium ion levels induced by the fatty acids in GPR120-expressing cells
}

\author{
Shin-ichi Adachi, Ai Eguchi, Kazuhiro Sakamoto, Hiroki Asano, Yasuko Manabe, Shigenobu Matsumura, \\ Satoshi Tsuzuki, Kazuo Inoue, and Tohru Fushiki \\ Laboratory of Nutrition Chemistry, Division of Food Science and Biotechnology, Graduate School of Agriculture, Kyoto University, \\ Kyoto 606-8502, Japan
}

(Received 29 August 2014; and accepted 10 September 2014)

\begin{abstract}
We recently reported that G-protein-coupled receptor 120 (GPR120) is expressed on taste buds, and that rodents showed preference for long-chain fatty acids (LCFA) at a low concentration. We also showed that the LCFA (1\% linoleic acid) increased the extracellular dopamine (DA) level in the nucleus accumbens (NAc), which participates in reward behavior. However, the mechanism underlying the involvement of the GPR120-agonistic activity of LCFA in the palatability of dietary fat remains elusive. Therefore, we examined the association between the GPR120-agonistic activity and palatability of LCFA. First, we measured $\mathrm{Ca}^{2+}$ signaling in HEK293 cells stably expressing GPR120 under stimulation by various LCFAs. We then assessed the palatability of the various LCFAs by testing the licking behavior in mice and measured the changes in the NAc-DA level by in vivo microdialysis. Consequently, 14- to 22-carbon unsaturated LCFAs showed strong GPR120-agonistic activity. Additionally, mice displayed high licking response to unsaturated 16and 18-carbon LCFAs, and unsaturated 18-carbon LCFA significantly increased the DA level. The licking rate and the LCFA-dependent increase in DA level also correlated well with the GPR120agonistic activity. These findings demonstrate that chemoreception of LCFA by GPR120 is involved in the recognition and palatability of dietary fat.
\end{abstract}

Most animals, including rodents, as well as humans prefer fat-rich foods $(3,23)$. The consumption of corn oil has been reported to produce a reward effect in mice, and the dopaminergic pathway in the nervous system has been implicated in the manifestation of this effect $(13,30)$. Additionally, sham and real feeding of corn oil were reported to induce the activation of the midbrain dopamine (DA) system, which is involved in reward behavior $(1,17)$. The mesolimbic system is thought to play a critical role in the reward effect, and the release of DA has been

Address correspondence to: Tohru Fushiki, PhD., Laboratory of Nutrition Chemistry, Division of Food Science and Biotechnology, Graduate School of Agriculture, Kyoto University, Kyoto 606-8502, Japan Tel: +81-75-753-6263, Fax: +81-75-753-6264

E-mail: tfushiki@kais.kyoto-u.ac.jp demonstrated when a natural and drug reward is acquired or when its acquisition is anticipated $(26,27)$. The midbrain dopaminergic circuits originate from the ventral tegmental area (VTA) and project to different sites, such as the nucleus accumbens (NAc), amygdala, and the prefrontal area, which are related to motivation, palatability, and addiction $(6,19,25)$. Iwakura et al. showed that the secretion of ghrelin, a hormone that stimulates food intake, is induced by DA in a ghrelin-producing cell line MGN3-1 (14). Since the extracellular concentration of DA in the NAc of the rat increases in a dose-dependent manner after self-administration of cocaine or consumption of sucrose, release of DA in the NAc could be considered as a form of index for the palatability or motivational drive $(9,21)$.

Recent studies have revealed that chemoreceptors of long-chain fatty acids (LCFAs) are involved in the 
recognition of fatty foods. We previously reported that, when fat was introduced into the oral cavity of rats, a certain percentage of triacylglycerides was hydrolyzed to LCFAs by lingual lipase (15). Gilbertson et al. demonstrated the regulation of $\mathrm{K}^{+}$channels in type II taste cells by unsaturated LCFAs and suggested that fatty acid chemoreceptors were present within taste cells (7). We found that CD36 fatty acid transporter was expressed on the apical side of taste cells in the circumvallate papillae (4). Additionally, CD36-deficient mice were reported to show a low taste preference for fat (16). Moreover, we reported that GPR120 was also expressed on the apical side of taste cells in the circumvallate papillae (18). The unsaturated LCFAs, such as oleic acid and linolenic acid, induced a rise in concentration of intracellular calcium ion $\left(\left[\mathrm{Ca}^{2+}\right]_{\mathrm{i}}\right)$ in Human Embryonic Kidney 293 (HEK293) cells stably expressing GPR120. On the other hand, LCFA esters and capric acid did not induce this increase in concentration (11). When the licking behavior of the mice was tested, it was found that the mice exhibited equally strong preference for a low concentration of linoleic acid as that for $100 \%$ corn oil (29). In addition, the mice exhibited a similar strong preference for LCFAs, such as oleic, linolenic, and linoleic acid, whereas they did not display any preference for LCFA esters nor long-chain fatty alcohols (28). Moreover, ingesting linoleic acid at a low concentration increased extracellular DA release in the NAc of rats (1). Compared with wild-type mice, GPR120 knock-out mice showed a lower preference for LCFAs and lower response of the chorda tympani and glossopharyngeal nerve to LCFAs (2). These findings lead us to postulate that, in the oral cavity, fat is hydrolyzed to LCFA by lingual lipase and that chemoreception of this LCFA by receptor proteins, such as CD36 and GPR120 expressed on the taste cells, could be involved in the oral recognition and palatability of fats. However, it remains unclear as to which structural characteristics of LCFA are responsible for the GPR120-agonistic activity and if these activities could be implicated in the palatability of LCFA.

Therefore, in this study, we examined the relationship between the GPR120-agonistic properties of ligands and the palatability of LCFAs. First, using HEK293 cells stably expressing human GPR120, we examined the effect of various LCFAs on the concentrationof $\left[\mathrm{Ca}^{2+}\right]_{\mathrm{i}}$ by using a fluorescence spectrophotometer. We then assessed the palatability for a variety of LCFAs at a low concentration by testing the licking behavior of the mice. Next, we studied the change in the extracellular concentration of DA in the NAc of the mice by in vivo microdialysis after ingestion of various low-concentration LCFAs. In both the tests, we used $0.0322 \mathrm{~mol} / \mathrm{L}$ fatty acid in mineral oil. The molar concentration was equal to a volume/volume $\%$ concentration of $1 \%$ linoleic acid. This concentration of fatty acid was in the range that could be released from fat by lingual lipase and had only $1 / 100$ th of the calorie content of fats of the same weight.

\section{MATERIALS AND METHODS}

$\left[\mathrm{Ca}^{2+}\right]_{i}$ analysis

Cell culture. HEK293 cells were maintained in Dulbecco's Modified Eagle Medium (DMEM) containing $10 \%$ fetal bovine serum (FBS) and $1 \%$ penicillinstreptomycin in a humidified, $5 \% \mathrm{CO}_{2}$ atmosphere at $37^{\circ} \mathrm{C}$. Human GPR120 cDNA from the lung was provided by Pharmafoods International Co., Ltd. (Kyoto, Japan).

Transfection into cells. Human GPR120 was transfected into HEK293 cells using the lipofection method as per the manufacturer's instructions. For the control, an empty vector was transfected into the HEK293 cells (Empty). Forty-eight hours after transfection, the medium was replaced with fresh medium containing $400 \mu \mathrm{g} / \mathrm{mL}$ G418 (Wako, Osaka, Japan) for the selection of the transfected cells, following which a single clone was selected by the standard limiting dilution method. GPR120 expression in cells was confirmed by reverse transcriptase polymerase chain reaction (RT-PCR). Transfected cells were maintained in DMEM containing 10\% FBS, $1 \%$ penicillin-streptomycin, and G-418 $(400 \mu \mathrm{g} / \mathrm{mL})$.

Reagents for $\left[\mathrm{Ca}^{2+}\right]_{i}$ analysis. All fatty acids-caprylic acid, C8:0; capric acid, C10:0; lauric acid, C12:0; myristic acid, C14:0; myristoleic acid, C14:1; palmitic acid, C16:0; palmitoleic acid, C16:1; stearic acid, C18:0; oleic acid, C18:1; linoleic acid, C18:2; linolenic acid, $\mathrm{C} 18: 3$; stearidonic acid, $\mathrm{C} 18: 4$; arachidic acid, C20:0; arachidonic acid, C20:4; eicosapentaenoic acid (EPA), C20:5; behenic acid, C22:0; docosahexaenoic acid (DHA), C22:6; methyl oleate, methyl linoleate, and methyl linolenate - were purchased from Sigma (St. Louis, MO, USA) and stored at $-20^{\circ} \mathrm{C}$ until use. All cell culture reagents (HEPES, Hanks buffer, DMEM, FBS, penicillin-streptomycin, and Lipofectamine) were purchased from Invitrogen (Carlsbad, CA, USA). All other chemicals, unless stated otherwise, were purchased from Sigma. 
$\mathrm{Ca}^{2+}$ mobilization assay. $\mathrm{Ca}^{2+}$ loading buffer comprised of $5 \mu \mathrm{L}$ Fluo-3AM $(1 \mu \mathrm{M}$; Dojindo, Kumamoto, Japan) and $10 \mu \mathrm{L}$ pluronic F-127 (Wako), diluted to yield $10 \mathrm{~mL} \mathrm{Ca}{ }^{2+}$ assay buffer $(20 \mathrm{mM}$ HEPES $\mathrm{pH}$ 7.6, $0.01 \%$ BSA, $1 \mathrm{mM}$ Probenecid [Wako] in Hanks solution) (12). On the day before the assay, $5 \times 10^{4}$ cells were seeded in 96-well, poly-D-lysinecoated plates (BD BioCoat, Franklin Lakes, NJ, USA). The cells were washed once with phosphate buffered saline (PBS) and incubated in a final volume of $100 \mu \mathrm{L} /$ well in $\mathrm{Ca}^{2+}$ loading buffer for $60 \mathrm{~min}$ at $37^{\circ} \mathrm{C}$. Then, the cells were washed twice with $\mathrm{Ca}^{2+}$ assay buffer and the assay was carried out in $100 \mu \mathrm{L}$ of $\mathrm{Ca}^{2+}$ assay buffer. Changes in $\mathrm{Ca}^{2+}$ levels were monitored by a fluorescence spectrophotometer (Powerscan HT, DS Pharma Biomedical Co., Ltd., Osaka, Japan) at an excitation wavelength of $485 \mathrm{~nm}$ and emission wavelength of $528 \mathrm{~nm}$. The maximum intracellular $\mathrm{Ca}^{2+}\left(\left[\mathrm{Ca}^{2+}\right]_{\mathrm{i}}\right)$ fluorescence intensity was obtained as the mean of triplicate assays. Test samples for the $\mathrm{Ca}^{2+}$ mobilization assay were prepared by sonication in $\mathrm{Ca}^{2+}$ buffer just prior to the assay.

\section{Behavioral test}

Animals. This study was conducted in accordance with the ethical guidelines of the Kyoto University Animal Experimentation Committee, in complete compliance with the National Institutes of Health Guide for the Care and Use of Laboratory Animals, and it was approved by the above-mentioned committee. Male BALB/c mice (Japan SLC, Hamamatsu, Japan) at 8 weeks of age were housed in plastic cages in a room with a 12-h light-dark cycle (dark phase of $18: 00-6: 00)$ and constant temperature $\left(24 \pm 1{ }^{\circ} \mathrm{C}\right)$. They were separately housed for $>5$ days for acclimatization to the environment. The animals were provided with tap water and regular MF mouse food (Oriental Yeast, Tokyo, Japan) ad libitum.

Materials. Corn oil was purchased from Ajinomoto (Tokyo, Japan) and mineral oil was purchased from Kaneda Company (Tokyo, Japan). All fatty acidscaproic acid, C6:0; caprylic acid, C8:0; capric acid, C10:0; lauric acid, C12:0; myristic acid, C14:0; myristoleic acid, C14:1; palmitic acid, C16:0; palmitoleic acid, C16:1; stearic acid, C18:0; oleic acid, C18:1 cis-9; elaidic acid, C18:1 trans-9; cis-vaccenic acid, C18:1 cis-11; trans-vaccenic acid, C18:1 trans-11; linoleic acid, C18:2; linolenic acid, C18:3; stearidonic acid, C18:4; arachidic acid, C20:0; arachidonic acid, C20:4; behenic acid, C22:0; docosa- hexaenoic acid (DHA), C22:6; lignoceric acid, C24:0; methyl oleate, methyl linoleate, and methyl linolenate-were purchased from Sigma (St. Louis, MO, USA). They were $99 \%$ pure, stored at $-20^{\circ} \mathrm{C}$ until use and then diluted in mineral oil to $0.0322 \mathrm{~mol} / \mathrm{L}$, which is equivalent to a $\mathrm{v} / \mathrm{v} \%$ concentration of $1 \%$ linoleic acid. Capric acid, lauric acid, myristic acid, palmitic acid, stearic acid, elaidic acid, trans-vaccenic acid, arachidic acid, behenic acid, and lignoceric acid do not dissolve at room temperature. Therefore, we heated each fatty acid solution to $75^{\circ} \mathrm{C}$ to eliminate the effects of difference in temperature. For testing the licking behavior, we used all the above fatty acids. In the microdialysis test, we used capric acid, lauric acid, stearic acid, oleic acid, linoleic acid, docosahexaenoic acid, and methyl linoleate. The other reagents were purchased from Nacalai Tesque (Kyoto, Japan).

\section{Evaluation of the licking behavior}

Apparatus for the test. Licking behavior was evaluated in a custom-made licking test chamber (Muromachi Kikai, Tokyo, Japan) previously described (22). In brief, the test chamber $(150 \times 120 \times 130$ $\mathrm{mm}$ ) was made of Plexiglas with an automatic shutter placed on the front wall, $1.5 \mathrm{~cm}$ above the metal-grid floor. When the shutter opened, mice gained access to a stainless steel drinking spout. The licking response was recorded by a computer. The licking rate was calculated for $60 \mathrm{~s}$ starting from the first lick. Given this very short period, we can rule out any contribution of post-ingestive feedback to the licking behavior.

Evaluation of the licking behavior. To allow the mice to be habituated to the test environment and to get accustomed to ingesting corn oil and mineral oil, they were kept in the test chamber for $30 \mathrm{~min}$ and offered corn oil and mineral oil for $30 \mathrm{~min}$. This training lasted until the mice could discriminate corn oil from mineral oil to the same degree as the previous report (29). After training, the mice were offered linoleic acid and stearic acid. We confirmed that the preference for linoleic acid was high and that for stearic acid was low, similar to the previous report (28). The licking behavior of the mice was then tested. In the test, the mice were offered the test fluids for 30 min once a day in the test chamber. We recorded the licking rate for $1 \mathrm{~min}$ from the first lick and the intake for $30 \mathrm{~min}$ from the start of presentation of the test fluid. To avoid order effects, each mouse was offered the test fluids in a different order. 


\section{Microdialysis test}

Training protocols for oil ingestion in the microdialysis test. To allow the mice to get accustomed to ingesting corn oil, mineral oil, and fatty acid fluid, the mice were fed these liquids in their cages before surgery and then in the microdialysis cage after recovery from surgery. The mice were deprived of water and food for $30 \mathrm{~min}$, and then the liquids were kept in front of the mice for $10 \mathrm{~min}$. Before surgery, the mice were presented with corn oil and mineral oil at the same time on days 1 and 2. To confirm their preference for corn oil over mineral oil, the mice were subjected to a 2-bottle preference test for corn oil vs. mineral oil on day 3 . The liquid bottles were positioned randomly. The mice were randomly presented with mineral oil, lauric acid, and linoleic acid on days 4 to 6 and capric acid, stearic acid, oleic acid, methyl linoleate, and docosahexaenoic acid on days 7 to 11 . After recovering from the surgery, the mice were presented with corn oil on day 1 and all other liquids in random order on days 2 to 9 in the microdialysis cage. The mice were then subjected to a microdialysis test on day 10 .

Microdialysis surgery. The animals were anesthetized with pentobarbital sodium (Nembutal; Dainippon Pharmaceutical Co., Tokyo, Japan) and placed in a stereotaxic frame modified for surgery in mice. The skulls of the mice were subsequently exposed and holes were drilled for microdialysis. The coordinates for the NAc guide cannula (AG-5; Eicom, Kyoto, Japan) were AP, 1.2; ML, 0.6; and DV, 3.2 from the bregma. The coordinates were determined according to the stereotaxic atlas of Paxinos and Franklin (20). The cannulas were secured to the skull with a LOCTITE 454 adhesive bond (Henkel Japan, Yokohama, Japan). A dummy AD-5 cannula (Eicom) was inserted into the guide cannula and secured with an AC-1 cap nut (Eicom). The mice were allowed 3 to 5 days to recover from the surgery. Each mouse implanted with a probe in the NAc was used for a single microdialysis procedure with a single test liquid.

Procedure. The experiments were conducted during the light period of the light-dark cycle.The dummy cannula was removed on the day of the experiment, and the AI-5-1.5 microdialysis probe (Eicom, $1.5 \mathrm{~mm}$ membrane length) was inserted into the NAc via the guide cannula. The mice were placed in the microdialysis cage at $8: 00 \mathrm{a} . \mathrm{m}$. for $3.5 \mathrm{~h}$ without food and water, and then presented with the test liquid at $11: 30$ p.m. for $10 \mathrm{~min}$. The amount of liquid ingest- ed was also recorded. The rats remained in the microdialysis cage for another $80 \mathrm{~min}$ after presenting the test liquid. Ringer's solution containing $147 \mathrm{mM}$ $\mathrm{Na}^{+}, 4 \mathrm{mM} \mathrm{K}^{+}, 2.3 \mathrm{mM} \mathrm{Ca}^{2+}$, and $155.6 \mathrm{mM} \mathrm{Cl}^{-}$was perfused at $3 \mu \mathrm{L} / \mathrm{min}$ by an ESP-64 micro-syringe pump (Eicom). Dialysate collection was started $30 \mathrm{~min}$ before the liquids were presented, and the collection conducted every $10 \mathrm{~min}$ for a total of 120 min thereafter. To quantify DA and 5-HT levels in the dialysate, samples were analyzed by reversedphase high performance liquid chromatography (HPLC) with an electrochemical detector, using an Eicompak PP-ODS II column (4.6 i.d. $\times 30 \mathrm{~mm}$ long; Eicom). The voltage applied was set at $400 \mathrm{mV}$ (relative to an $\mathrm{Ag} / \mathrm{AgCl}$ reference electrode). The mobile phase at a flow rate of $500 \mu \mathrm{L} / \mathrm{min}$ consisted of a $98 \%(\mathrm{v} / \mathrm{v}) 0.1 \mathrm{M}$ phosphate buffer at $\mathrm{pH} 6.0,2 \%$ (v/v) methanol, $500 \mathrm{mg} / \mathrm{L}$ sodium decane sulfate, and $50 \mathrm{mg} / \mathrm{L}$ EDTA-2Na. The mean value obtained from 3 samples from -30 to -10 min was set as the $100 \%$ baseline level, and all subsequent sample values were expressed as a percentage of the baseline value.

Histological analysis. Upon completion of the experiment, the mice were deeply anesthetized with sodium pentobarbital. The brain was removed from the skull, frozen, and cut into $20-\mu \mathrm{m}$ sections. The placement of the microdialysis probe was verified by thionine blue staining. Data obtained from the mice with inappropriate probe placement were excluded from the analysis.

Statistics. Data are expressed as the mean \pm SEM. Data from $\left[\mathrm{Ca}^{2+}\right]_{\mathrm{i}}$ assay were analyzed using a oneway ANOVA and Dunnett's post hoc test. Data obtained by testing the licking behavior were analyzed using one-way repeated ANOVA and Dunnett's post hoc test. Data from the 2-bottle preference test were analyzed by a paired $t$-test. Changes in DA and 5-HT levels were compared with the corresponding baseline value by one-way repeated ANOVA and Tukey's multiple-comparison test as a post-hoc test. Mean differences among the 3 groups at each time point were analyzed by two-way repeated-measures ANOVA and Bonferroni's multiple-comparison as a post-hoc test. The amount of each fluid ingested during microdialysis was analyzed by a one-way ANOVA and Dunnett's test as a post-hoc test. Correlation coefficient was obtained by Pearson correlation test. $P$ values of $5 \%$ or less were considered statistically significant. Statistical analyses were conducted by using the Prism 6 software package 
(GraphPad, San Diego, CA, USA).

\section{RESULTS}

Intracellular $\mathrm{Ca}^{2+}$ Assay in HEK293 cells

Fig. 1 shows the maximum intracellular $\mathrm{Ca}^{2+}$ fluorescence intensity in the response induced by $10 \mu \mathrm{M}$ of different fatty acids. Intracellular calcium levels in the response induced by myristic acid (C14:0), myristoleic acid (C14:1), palmitic acid (C16:0), palmitoleic acid (C16:1), oleic acid (C18:1), linoleic acid (C18:2), linolenic acid (C18:3), stearidonic acid (C18:4), arachidonic acid (C20:4), eicosapentaenoic acid (C20:5), and docosahexaenoic acid (C22:6) were higher than those induced by the buffer (versus buffer by Dunnett's test: $P<0.01$ for palmitic acid; $P<0.001$ for other fatty acids). On the other hand, the level of response induced by caprylic acid (C8:0), capric acid (C10:0), lauric acid (C12:0), stearic acid (C18:0), methyl oleate $\left(\mathrm{C} 18: 1-\mathrm{CH}_{3}\right)$, methyl linoleate $\left(\mathrm{C} 18: 2-\mathrm{CH}_{3}\right)$, methyl linolenate $\left(\mathrm{C} 18: 3-\mathrm{CH}_{3}\right)$, arachidic acid (C20:0), and behenic acid (C22:0) were not significant.

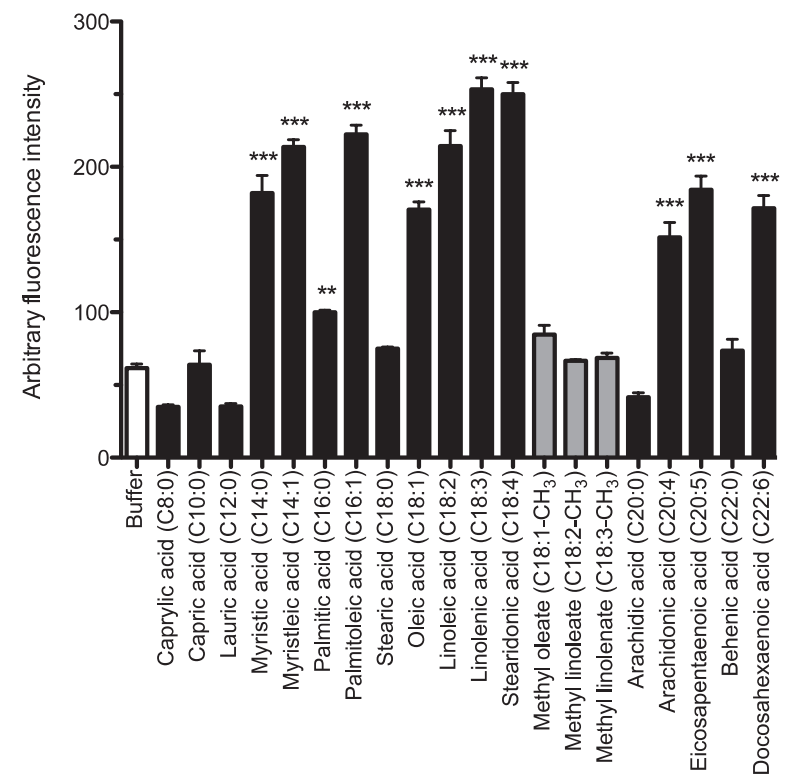

Fig. 1 Intracellular $\mathrm{Ca}^{2+}\left(\left[\mathrm{Ca}^{2+}\right]_{\mathrm{i}}\right)$ level increases induced by $10 \mu \mathrm{M}$ of various fatty acids in HEK293 cells stably expressing GPR120. $\left[\mathrm{Ca}^{2+}\right]_{\mathrm{i}}$ response induced by $10 \mu \mathrm{M}$ test fatty acid in HEK293 cells GPR120 was monitored by a fluorescence spectrophotometer for $1 \mathrm{~min}$ at an excitation wavelength of $485 \mathrm{~nm}$ and an emission wavelength of 528 $\mathrm{nm}$. $\left[\mathrm{Ca}^{2+}\right]_{\mathrm{i}}$ level was expressed as the maximum fluorescence intensity observed in $1 \mathrm{~min}$. Data are presented as the mean \pm SEM $\left(\mathrm{n}=3,{ }^{\star \star} P<0.01,{ }^{\star \star \star} P<0.001\right.$, versus control buffer).

\section{Evaluation of the licking behavior}

Fig. 2 shows the initial licking rate with various fatty acids. The mice exhibited a significantly higher licking rate with palmitoleic acid (C16:1), oleic acid (C18:1 cis-9), linoleic acid (C18:2), linolenic acid (C18:3), stearidonic acid (C18:4), arachidonic acid (C20:4), and docosahexaenoic acid (C22:6) than with mineral oil (versus mineral oil by Dunnett's test: $P<0.01$ for arachidonic acid; $P<0.001$ for other fatty acids). On the other hand, the mice did not respond significantly to caproic acid (C6:0), caprylic acid (C8:0), capric acid (C10:0), lauric acid (C12:0), myristic acid (C14:0), myristoleic acid (C14:1), palmitic acid (C16:0), stearic acid (C18:0), elaidic acid (C18:1 trans-9), cis-vaccenic acid (C18:1 cis-11), trans-vaccenic acid (C18:1 trans-11), methyl oleate $\left(\mathrm{C} 18: 1-\mathrm{CH}_{3}\right)$, methyl linoleate $\left(\mathrm{C} 18: 2-\mathrm{CH}_{3}\right)$, methyl linolenate, $\left(\mathrm{C} 18: 3-\mathrm{CH}_{3}\right)$, arachidic acid $(\mathrm{C} 20: 0)$, behenic acid (C22:0), and lignoceric acid (C24:0).

Similar to the result for the licking rate, the intake amount for palmitic acid (C16:0), palmitoleic acid (C16:1), oleic acid (C18:1 cis-9), elaidic acid (C18:1 trans-9), linoleic acid (C18:2), cis-vaccenic acid (C18:1 cis-11), linolenic acid (C18:3), and stearidonic acid (C18:4) were significantly greater than that of mineral oil (versus mineral oil by Dunnett's



Fig. 2 Initial licking rate with various fatty acids at a concentration of $0.0322 \mathrm{~mol} / \mathrm{L}$ in the test performed to evaluate licking behavior. Mice $(n=15)$ were offered a bottle of the test fluid and the licking rate over the first $60 \mathrm{~s}$ was recorded as the initial licking rate. Data are presented as the mean \pm SEM $\left({ }^{\star *} P<0.01,{ }^{* \star} P<0.001\right.$, versus initial licking rate with mineral oil). 
test: $P<0.01$ for palmitic acid and elaidic acid; $P<0.001$ for other fatty acids) (Table 1 ). However, the intake amount for all other fatty acids was not significant.

\section{Microdialysis test}

\section{Validation of the preference for oil after ingestion training}

The 2-bottle preference test on day 3 before surgery demonstrated that the mice significantly preferred corn oil to mineral oil (Fig. 3, $P<0.05$ by the paired $t$-test).

\section{Effect of intake on the extracellular DA and 5-HT} level in the NAc

Fatty acid intake in the microdialysis test. The intake of oleic acid and linoleic acid was greater than that of mineral oil during the microdialysis test (Fig. 4; $P<0.05$ vs. mineral oil by Dunnett's test). The intake of corn oil, caprylic acid, lauric acid, stearic acid, methyl linoleate, and docosahexaenoic acid

Table 1 Intake amounts for various types of fatty acid in the licking test for $30 \mathrm{~min}$

\begin{tabular}{|c|c|c|}
\hline Fatty acid & $\begin{array}{l}\text { Carbon } \\
\text { chain length }\end{array}$ & $\begin{array}{l}\text { Intake } \\
(\mathrm{g} / 30 \mathrm{~min})\end{array}$ \\
\hline Mineral oil & & $0.02 \pm 0.01$ \\
\hline Caproic acid & $\mathrm{C} 6: 0$ & $0.03 \pm 0.01$ \\
\hline Caprylic acid & $\mathrm{C} 8: 0$ & $0.07 \pm 0.05$ \\
\hline Capric acid & $\mathrm{C} 10: 0$ & $0.30 \pm 0.18$ \\
\hline Lauric acid & $\mathrm{C} 12: 0$ & $0.10 \pm 0.06$ \\
\hline Myristic acid & $\mathrm{C} 14: 0$ & $0.42 \pm 0.16$ \\
\hline Myristleic acid & $\mathrm{C} 14: 1$ & $0.31 \pm 0.14$ \\
\hline Palmitic acid & $\mathrm{C} 16: 0$ & $0.74 \pm 0.22 * *$ \\
\hline Palmitoleic acid & $\mathrm{C} 16: 1$ & $1.84 \pm 0.19 * * *$ \\
\hline Stearic acid & $\mathrm{C} 18: 0$ & $0.06 \pm 0.04$ \\
\hline Oleic acid & C18:1 & $1.51 \pm 0.27 * * *$ \\
\hline Elaidic acid & $\mathrm{C} 18: 1$ & $0.80 \pm 0.24^{* *}$ \\
\hline cis-Vaccenic acid & $\mathrm{C} 18: 1$ & $0.74 \pm 0.23 * *$ \\
\hline trans-Vaccanic acid & $\mathrm{C} 18: 1$ & $0.56 \pm 0.18$ \\
\hline Linoleic acid & $\mathrm{C} 18: 2$ & $2.28 \pm 0.10 * * *$ \\
\hline Linolenic acid & $\mathrm{C} 18: 3$ & $1.53 \pm 0.13 * * *$ \\
\hline Stearidonic acid & $\mathrm{C} 18: 4$ & $1.07 \pm 0.17 * * *$ \\
\hline Methyl oleate & $\mathrm{C} 18: 1-\mathrm{CH}_{3}$ & $0.27 \pm 0.11$ \\
\hline Methyl linoleate & $\mathrm{C} 18: 2-\mathrm{CH}_{3}$ & $0.19 \pm 0.10$ \\
\hline Methyl linolenate & $\mathrm{C} 18: 3-\mathrm{CH}_{3}$ & $0.20 \pm 0.07$ \\
\hline Arachidic acid & $\mathrm{C} 20: 0$ & $0.07 \pm 0.02$ \\
\hline Arachidonic acid & $\mathrm{C} 20: 4$ & $0.19 \pm 0.04$ \\
\hline Behenic acid & $\mathrm{C} 22: 0$ & $0.09 \pm 0.05$ \\
\hline Docosahexaenoic acid & $\mathrm{C} 22: 6$ & $0.33 \pm 0.07$ \\
\hline Lignoceric acid & $\mathrm{C} 24: 0$ & $0.05 \pm 0.01$ \\
\hline
\end{tabular}

Values are means $\pm \operatorname{SEM}(\mathrm{n}=15) . * * P<0.01, * * * P<0.001$, vs. mineral oil. was not significant.

Time-course change in the DA level in the mouse $N A c$. Fig. 5A shows the time-course changes in DA level in the NAc of mice that had ingested each liquid. There was no difference in the baseline extracellular DA concentrations in the NAc (Table 2). There were no significant changes to DA levels in

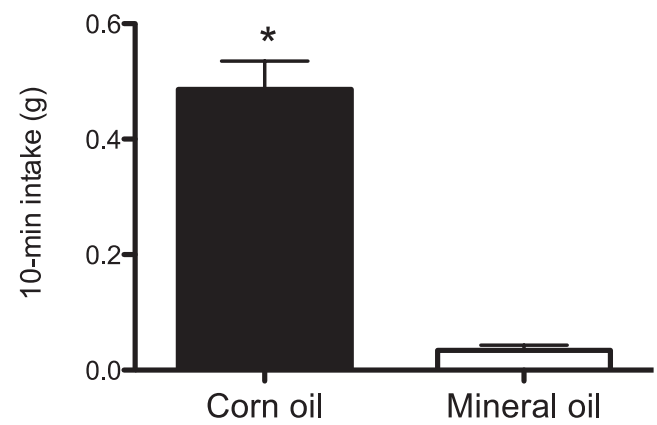

Fig. 3 Preference for corn oil before surgery. Mice $(n=48)$ were subjected to a 2-bottle choice test with the presentation of $100 \%$ corn oil and $100 \%$ mineral oil at the same time for $10 \mathrm{~min}$, and the amount of each liquid ingested was recorded. Data are presented as the mean intake \pm SEM summed for 10 min per mouse $(P<0.0001$, corn oil vs. mineral oil intake by a paired $t$-test).

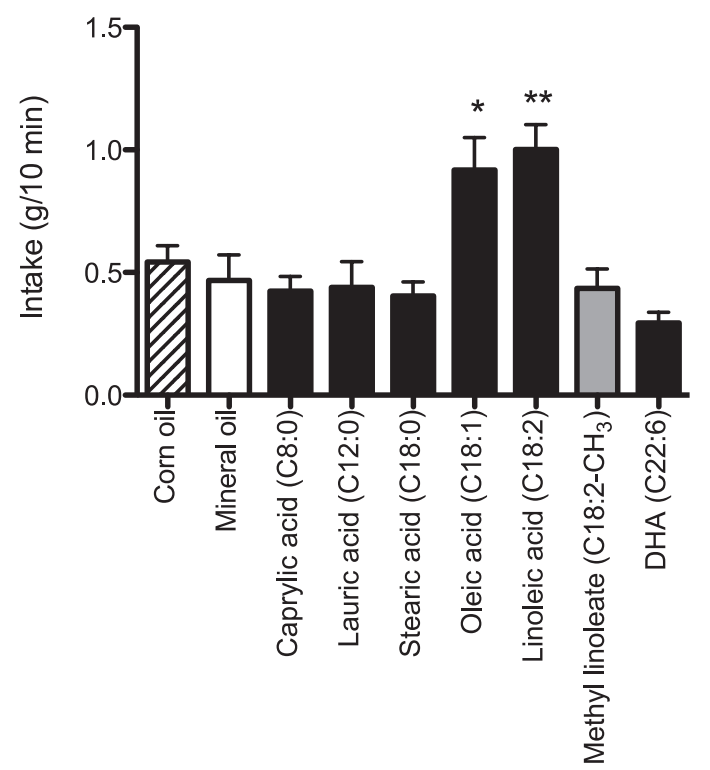

Fig. 4 Mean intake of each liquid per mouse with the 10min presentation during the microdialysis session. Each mouse was presented with the test liquids $(n=6$ for corn oil; $n=5$ for mineral oil; $n=5$ for capric acid; $n=5$ for lauric acid; $n=5$ for stearic acid; $n=6$ for oleic acid; $n=6$ for linoleic acid; $n=5$ for methyl linoleate; $n=5$ for DHA) at 0 min for $10 \mathrm{~min}$. Data are presented as the mean intake \pm SEM ( ${ }^{\star} P<0.05 ;{ }^{\star \star} P<0.01$, versus mineral oil intake). 
the mice ingesting mineral oil, capric acid (C10:0), lauric acid (C12:0), stearic acid (C18:0), methyl linoleate $\left(\mathrm{C} 18: 2-\mathrm{CH}_{3}\right)$, or docosahexaenoic acid (C22:6); however, the DA level in the mice ingesting corn oil was higher than baseline value at 0 to 20 min (versus baseline by Tukey's multiple-comparison test: $142.3 \pm 4.7 \%$ at $0 \mathrm{~min}, P<0.05 ; 147.3$ $\pm 9.8 \%$ at $10 \mathrm{~min}, P<0.05 ; 142.7 \pm 14.2 \%$ at $20 \mathrm{~min}$, $P<0.05)$. The DA level in the mice ingesting oleic acid (C18:1) was significantly higher than baseline value at times of 0 to $10 \mathrm{~min}$ (versus baseline: 132.3 $\pm 6.4 \%$ at $0 \mathrm{~min}, P<0.05 ; 140.8 \pm 2.6 \%$ at $10 \mathrm{~min}$, $P<0.01)$. The DA level in the mice ingesting linoleic acid was significantly higher than baseline val- ue at time point from 0 to $10 \mathrm{~min}$ (versus baseline: $148.0 \pm 10.7 \%$ at $0 \mathrm{~min}, P<0.05$ ). The DA level in the mice ingesting corn oil was significantly higher than that in the mice ingesting mineral oil at $10 \mathrm{~min}$ by Bonferroni's multiple-comparison test (versus mineral oil: $P<0.05$ ). The DA level in the mice ingesting linoleic acid (C18:2) was significantly higher than that in the mice ingesting mineral oil at 0 min by Bonferroni's multiple-comparison test (versus mineral oil: $P<0.05$ ). The DA levels in the mice ingesting capric acid, lauric acid, stearic acid, oleic acid, methyl linoleate, and docosahexaenoic acid were not significantly higher than that in the mice ingesting mineral oil. At the time when the
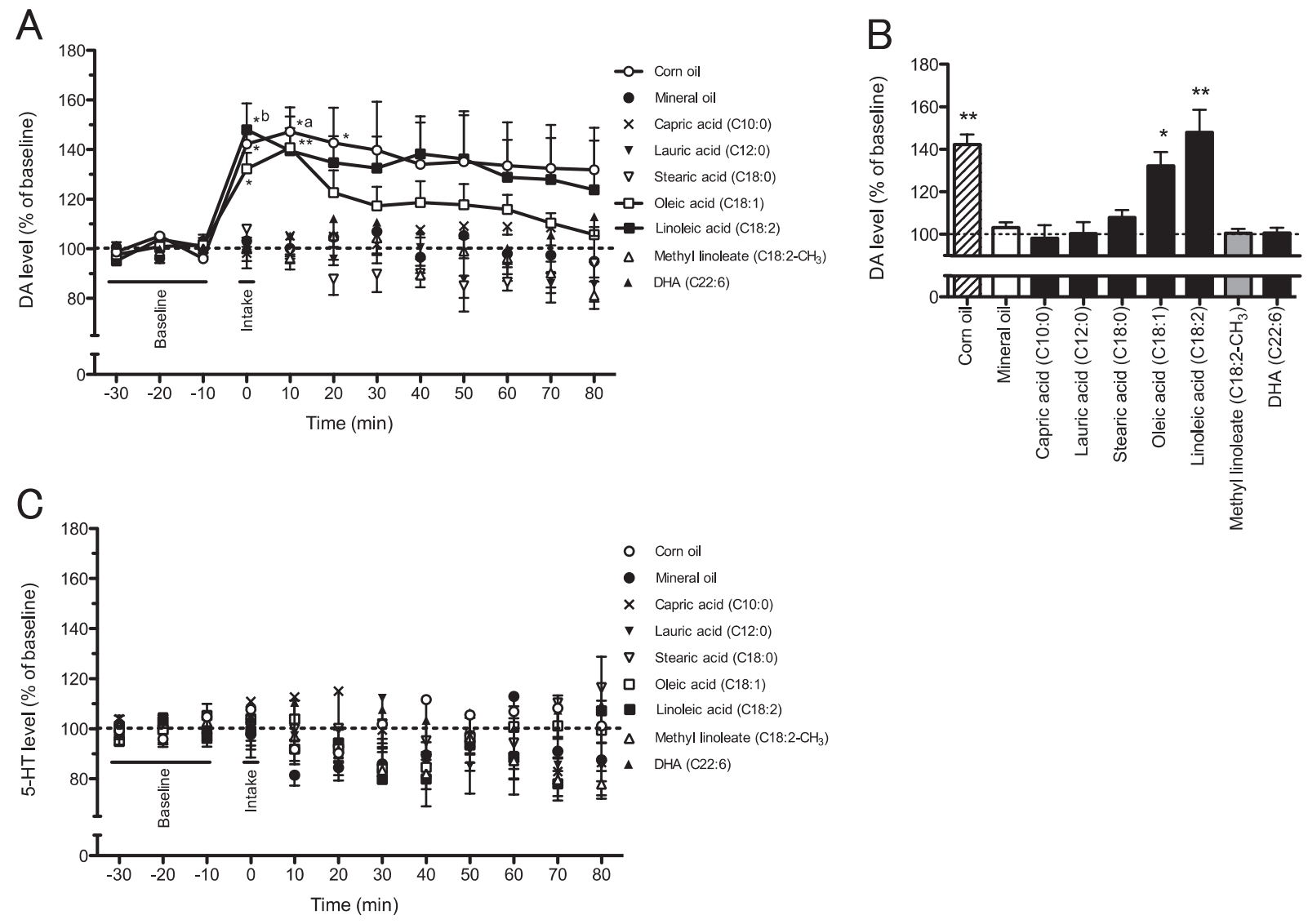

Fig. 5 Time-course change in the extracellular dopamine (DA) and serotonin (5-HT) levels in the mouse nucleus accumbens (NAc) during ingestion of the test liquids (100\% corn oil, $100 \%$ mineral oil, and $0.0322 \mathrm{~mol} / \mathrm{L}$ fatty acid) by mice. Each mouse was presented with the test liquids at $0 \mathrm{~min}$ for $10 \mathrm{~min}$, all subsequent sample values being expressed as a percentage of this baseline value, and as the mean \pm SEM at each time point. A, Time-course change of DA in the NAc is shown after ingesting the liquid ( $n=6$ for corn oil; $n=5$ for mineral oil; $n=5$ for capric acid; $n=5$ for lauric acid; $n=5$ for stearic acid; $n=6$ for oleic acid; $n=6$ for linoleic acid; $n=5$ for methyl linoleate; $n=5$ for DHA). Asterisks ( ${ }^{\star}$ ) represents significant increase from the baseline value $\left({ }^{\star} P<0.05 ;{ }^{* \star} P<0.01\right)$. Lowercase letters shows the statistical significance of the difference from the value for the mineral oil group at corresponding time points (a, for corn oil, $P<0.05$; $b$, for linoleic acid, $P<0.05)$. B, DA levels within the duration of 0 to 10 min during presentation of the liquid $\left({ }^{\star} P<0.05 ;{ }^{*} P<0.01\right.$, versus the mineral oil group). C, Time-course change of $5-\mathrm{HT}$ in the NAc is shown after ingesting the liquid $(n=5$ for corn oil; $n=3$ for mineral oil; $n=4$ for capric acid; $n=3$ for lauric acid; $n=4$ for stearic acid; $n=6$ for oleic acid; $n=4$ for linoleic acid; $n=3$ for methyl linoleate; $n=3$ for DHA). 


\begin{tabular}{|c|c|c|}
\hline & $\begin{array}{l}\text { DA concentration } \\
(\mathrm{ng} / \mu \mathrm{L})\end{array}$ & $\begin{array}{l}\text { 5-HT concentration } \\
(\mathrm{ng} / \mu \mathrm{L})\end{array}$ \\
\hline Corn oil & $0.037 \pm 0.005$ & $0.023 \pm 0.002$ \\
\hline Mineral oil & $0.042 \pm 0.018$ & $0.022 \pm 0.003$ \\
\hline Capric acid & $0.051 \pm 0.019$ & $0.029 \pm 0.003$ \\
\hline Lauric acid & $0.047 \pm 0.018$ & $0.024 \pm 0.005$ \\
\hline Stearic acid & $0.031 \pm 0.012$ & $0.026 \pm 0.003$ \\
\hline Oleic acid & $0.049 \pm 0.020$ & $0.022 \pm 0.003$ \\
\hline Linoleic acid & $0.043 \pm 0.018$ & $0.030 \pm 0.011$ \\
\hline Methyl linoleate & $0.047 \pm 0.018$ & $0.023 \pm 0.003$ \\
\hline Docosahexaenoic acid & $0.052 \pm 0.014$ & $0.023 \pm 0.003$ \\
\hline
\end{tabular}

Values are means \pm SEM $(n=5-6$ for DA; $n=3-6$ for $5-H T)$.

mice was ingesting fluid (at $0 \mathrm{~min}$ ), DA levels in the mice presented with corn oil, oleic acid, and linoleic acid were higher than that in the mice presented with mineral oil (Fig. 5B; versus mineral oil by Dunnett's test: $P<0.01$ for corn oil and linoleic acid; $P<0.05$ for oleic acid).

Time-course change in the 5-HT level in the mouse NAc. Fig. $5 \mathrm{C}$ shows the time-course changes in the 5-HT level in the NAc of mice that had ingested each liquid. There was no difference in the baseline extracellular 5-HT concentrations in the NAc (Table 2). There were no significant changes to 5-HT levels in the mice ingesting each fluid. Fig. 6 shows the placement of all the microdialysis probes into the NAc.

Correlations between $\mathrm{Ca}^{2+}$ fluorescence changes and initial licking rate between, and accumbens dopamine level

Fig. 7A shows a positive correlation between initial licking rate and the consumption of the different fatty acids in mice and maximum intracellular $\mathrm{Ca}^{2+}$ level estimated in cultured cells induced by corresponding fatty acid (Pearson correlation; $r=0.8005$, $P<0.0001, \mathrm{n}=19$ ). Additionally, there was a positive correlation between the $30 \mathrm{~min}$ intake amounts for fatty acids and $\left[\mathrm{Ca}^{2+}\right]_{\mathrm{i}}$ levels (data not shown; Pearson correlation; $r=0.7308, P=0.0004, \mathrm{n}=19$ ). Fig. 7B shows a positive correlation between cumulative value of DA levels in the NAc at 0 to $80 \mathrm{~min}$ in the mice offered various types of fatty acid and $\left[\mathrm{Ca}^{2+}\right]_{\mathrm{i}}$ levels (Pearson correlation; $r=0.8688, P=$ $0.0111, \mathrm{n}=7)$. Additionally, there was a positive correlation between DA levels at the time ( 0 to $10 \mathrm{~min}$ ) when the mice were offered the fluids and $\left[\mathrm{Ca}^{2+}\right]_{\mathrm{i}}$ levels (data not shown; Pearson correlation; $r=$

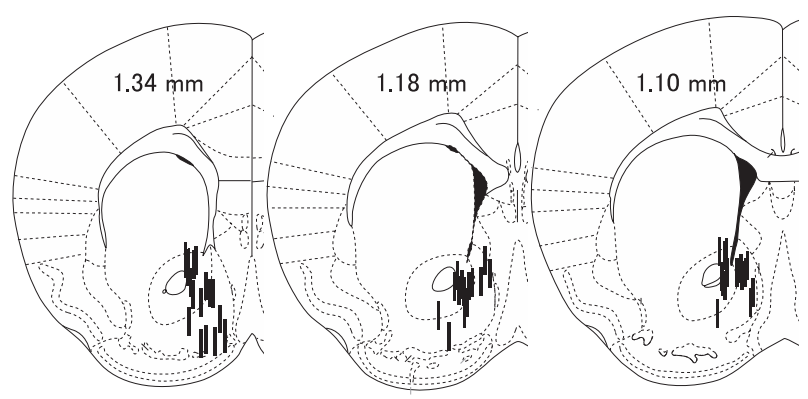

Fig. 6 Placement of all the microdialysis probes into the NAc. Black bar represents the tip of the probe. The number on each section is the distance in millimeters anterior from the bregma according to the stereotaxic atlas of Paxinos and Franklin (20).

0.7829, $P=0.0374, \mathrm{n}=7)$.

\section{DISCUSSION}

Intracellular $\mathrm{Ca}^{2+}$ assay

Since there was no response to fatty acids in the HEK293 cells transfected with an empty vector, it was confirmed that the fluorescence changes stimulated by fatty acids were the GPR120-specific responses (data not shown).

The addition of saturated and unsaturated 14- and 16-carbon fatty acids induced a significantly higher increase in $\left[\mathrm{Ca}^{2+}\right]_{\mathrm{i}}$ than that of the control buffer in the HEK293 cells stably expressing GPR120. Additionally, in the 18-, 20-, and 22-carbon fatty acids, increases in $\left[\mathrm{Ca}^{2+}\right]_{\mathrm{i}}$ induced by unsaturated fatty acids were significantly higher than those induced by control buffer, whereas saturated forms of these fatty acids caused no significant response. These results demonstrate that saturated 14- and 16-carbon fatty acids and unsaturated 14-, 16-, 18-, 20-, and 
A

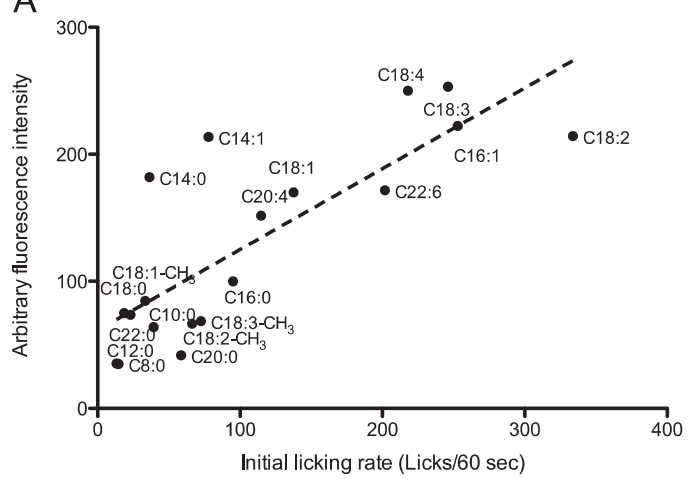

B

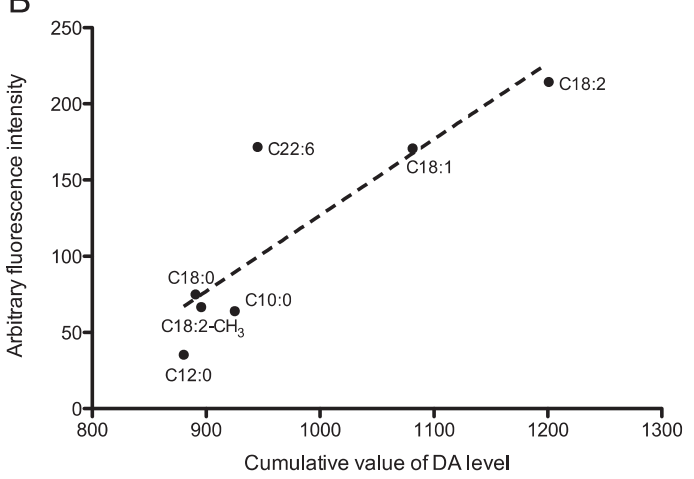

Fig. 7 Correlations of the maximum intracellular $\mathrm{Ca}^{2+}\left(\left[\mathrm{Ca}^{2+}\right]_{i}\right)$ fluorescence intensities and the initial licking rates $(A)$, and of the maximum $\left[\mathrm{Ca}^{2+}\right]_{\mathrm{i}}$ fluorescence intensities and the cumulative values of DA level (B). A, $X$ axis shows the averaged initial licking rate with various fatty acids and $\mathrm{Y}$ axis shows the averaged maximum $\left[\mathrm{Ca}^{2+}\right]_{\mathrm{i}}$ fluorescence intensities in the response induced by corresponding fatty acid (Pearson correlation; $r=0.7421, P=0.0002, \mathrm{n}=19$ ). $\mathrm{B}, \mathrm{X}$ axis shows the cumulative value of averaged DA level at 0 to $80 \mathrm{~min}$ in the NAc of mice after ingesting various fatty acids and $Y$ axis shows the averaged maximum $\left[\mathrm{Ca}^{2+}\right]_{i}$ fluorescence intensities in the response induced by corresponding fatty acid (Pearson correlation; $r=0.8688, P=0.0111, \mathrm{n}=7$ ).

22-carbon fatty acids are strong ligands for GPR120. Moreover, in 18- and 20-carbon fatty acids, $\left[\mathrm{Ca}^{2+}\right]_{\mathrm{i}}$ levels tended to increase due to poly unsaturated fatty acids like stearic acid (C18:0), oleic acid (C18:1), linoleic acid (C18:2), linolenic acid (C18:3), stearidonic acid (C18:4), arachidic acid (C20:0), arachidonic acid (C20:4), and EPA (C20:5). This result shows that the structure of poly unsaturated fatty acids is more suitable for GPR120 ligands in the 18- and 20-carbon acids than in other fatty acids. Further, $\left[\mathrm{Ca}^{2+}\right]_{i}$ levels stimulated by methyl esters of unsaturated 18-carbon fatty acid were not significant when compared with that of control. This result implies that the terminal carboxyl group of the fatty acids plays an important role in the recognition of fatty acid structure by GPR120. The results of $\left[\mathrm{Ca}^{2+}\right]_{i}$ assay in this study correspond well with previous reports by Hirasawa et al. and Galindo et al. $(5,11)$.

\section{Evaluation of licking behavior}

During the evaluation of the licking behavior with middle-chain fatty acids such as 6-carbon caproic acid, 8-C caprylic acid, and 10-C capric acid, as well as shorter-chain LCFAs such as 12-C lauric acid, 14-C myristic acid, and myristoleic acid, the initial licking rates were not significantly different from those observed by using mineral oil. On the other hand, in the test with longer LCFAs such as 16-C palmitoleic acid, 18-C oleic acid, linoleic acid, linolenic acid, stearidonic acid, 20-C arachidonic acid, and 22-C DHA, the initial licking rates were significantly higher than that with mineral oil. This result suggests that carbon chain length is involved in the palatability of fatty acid. In addition, since the mice displayed high licking response to unsaturated LCFAs (palmitic acid, 16:1; oleic acid, 18:1; linoleic acid, 18:2; linolenic acid, 18:3; stearidonic acid, 18:4; arachidonic acid, 20:4; and DHA, 22:6) and low licking response to saturated LCFAs (palmitic acid, 16:0; stearic acid, 18:0; arachidic acid, 20:0; and behenic acid, 22:0), these results indicate that palatability of fatty acids is affected by the saturation state of the fatty acid. Moreover, the mice showed a significantly higher licking rate with oleic acid, linoleic acid, and linolenic acid. On the other hand, the mice did not respond significantly to corresponding methyl esters, namely, methyl oleate, methyl linoleate, and methyl linolenate. Yoneda et al. reported that the mice exhibited low palatability for not only fatty acid ester but also fatty alcohol (28). These results imply that terminal carboxyl group of fatty acids is involved in their palatability. Further, the licking rate with oleic acid (cis-9-octadecenoic acid) was significantly higher than that with mineral oil. However, there was no difference in the licking rates with geometric isomer of oleic acid, elaidic acid (trans-9-octadecenoic acid) and mineral oil. Additionally, the licking rates with cis-vaccenic acid (cis-11-octadecenoic acid) and trans-vaccenic acid (trans-11-octadecenoic acid) which are regioisomers of oleic acid and elaidic acid, respectively, had no such effect. The results show that conformation of the fatty acid is important in the manifestation of palatability, in at least C18:1 fatty acids.

In this study, we observed that the amount of fat- 
ty acid intake over 30 min did not necessarily correlate with the initial licking rate. For example, the licking rates of myristoleic acid and elaidic acid were not significantly higher than that of mineral oil; however, their intake was greater than that of mineral oil. A possible explanation for this difference is that the post-ingestive effects of these acids may have promoted their consumption. In contrast, the licking rate of DHA was significantly higher than that of mineral oil, while the intake amount was not. Harden et al. reported that DHA stimulated significantly more release of cholecystokinin (CCK) than other LCFAs such as linoleic acid and oleic acid in secretin tumor (STC-1) cells (10). Therefore, release of CCK from the duodenum, which is suppressed after DHA ingestion, may suppress the overall intake of this fatty acid over $30 \mathrm{~min}$.

\section{Change in DA level in microdialysis test}

Previously, we have reported that extracellular DA level in the NAc of rats was elevated by ingesting $1 \%(\mathrm{v} / \mathrm{v})$ linoleic acid, which had a very low calorie level (approximately $0.09 \mathrm{kcal} / \mathrm{g}$ ), as compared to the similar increase observed with as high as $100 \%$ corn oil $(9 \mathrm{kcal} / \mathrm{g})$ ingestion (1). This showed that the reward value of fat, at least as estimated from the increase in DA level in the NAc, was determined by the chemoreception of LCFAs in the oral cavity, not by its caloric density. Similarly, in this study using mice, $100 \%$ corn oil and $1 \%$ linoleic acid significantly increased the NAc DA levels. Oleic acid also resulted in a significant increase in DA level. On the other hand, capric acid, lauric acid, stearic acid, methyl linoleate, and DHA caused no significant change in the DA levels. This result suggests that 18-carbon length, saturated state of fatty acid, and terminal carboxyl group are important for the rise in DA level observed upon the ingestion of these LCFAs. Further, the intake amount of oleic acid and linoleic acid during the 10 min of presentation in the microdialysis test was significantly greater than that of mineral oil, whereas other fatty acids presented in the microdialysis test showed no difference in consumption than that of mineral oil. These results indicated that the characteristics of fatty acids that caused an increase in the DA level corresponded well with the palatability of LCFAs.

\section{Correlation between $\left[\mathrm{Ca}^{2+}\right]_{i}$ level, licking rate, and DA level}

In this study, the GPR120-agonistic activity of LCFA as estimated in cell culture correlated with the initial licking rate and the increase in DA level. This sug- gests that the chemoreception of LCFA via GPR120 is the first step in the manifestation of this effect. $\left[\mathrm{Ca}^{2+}\right]_{\mathrm{i}}$ levels stimulated by myristic acid and myristoleic acid were higher than those achieved by using the control buffer, and the mice did not show significantly higher licking response to these fatty acids as compared to mineral oil. This difference may be attributed to the influence of the flavors in these fatty acids, which may have resulted in the mice avoiding their consumption. Godinot et al. reported that non-fatty acid agonists of GPR120 activated the glossopharyngeal nerve of mice and that the mice did not show any preference for non-fatty acid agonists of GPR120 (8). These findings imply that the palatability of LCFAs at low concentration may not consist of only GPR120 and that composition of the palatability may include other mechanisms such as chemoreception via CD36 and GPR40. Both of CD36 and GPR40 are reported to be expressed in the taste cell, while GPR120 is reported to be involved in the recognition and palatability of fat in the oral cavity (24). Further studies using GPR120 knockout mice are necessary to determine whether the expression of GPR120 is implicated in the palatability of fat and increase in DA level when ingesting fat. The agonistic activity of receptor proteins expressed in the oral cavity, such as CD36 and GPR40 requires investigation and a detailed study is warranted, on the relationship between agonistic activity and palatability using animal models.

To summarize, we found that saturated 14- and 16-carbon fatty acids and unsaturated 14-, 16-, 18-, 20-, and 22-carbon fatty acids are strong ligands for GPR120 that cause an increase in $\left[\mathrm{Ca}^{2+}\right]_{i}$ levels in the cultured cells. We also revealed that mice have high palatability for unsaturated 16-, 18-, 20-, and 22 -carbon fatty acids. Moreover, DA level in the NAc of mice was elevated after ingestion of unsaturated 18-carbon acid to the same extent as $100 \%$ corn oil. Further, the agonistic activity of GPR 120 ligands correlated with the palatability of LCFAs in the oral cavity and the reward values based on DA levels in the NAc. These findings suggest that the chemoreception of LCFAs via GPR120 is implicated in the palatability of LCFAs, and they support the notion that the chemoreception of LCFAs released from fat by lingual lipase plays an important role in the detection of fat in the oral cavity as well as fat palatability, including the reward effect.

\section{Acknowledgements}

The authors would like to thank Pharmafoods Inter- 
national Co., Ltd for providing GPR120 cDNA. This work was supported by the Science and Technology Research Promotion Program for agriculture, forestry, fisheries, and food industry.

\section{REFERENCES}

1. Adachi S, Endo Y, Mizushige T, Tsuzuki S, Matsumura S, Inoue K and Fushiki T (2013) Increased levels of extracellular dopamine in the nucleus accumbens and amygdala of rats by ingesting a low concentration of a long-chain fatty acid. Biosci Biotechnol Biochem 77, 2175-2180.

2. Cartoni C, Yasumatsu K, Ohkuri T, Shigemura N, Yoshida R, Godinot N, le Coutre J, Ninomiya Y and Damak S (2010) Taste preference for fatty acids is mediated by gpr40 and gpr120. J Neurosci 30, 8376-8382.

3. Drewnowski A and Greenwood MR (1983) Cream and sugar: human preferences for high-fat foods. Physiol Behav 30, 629-633.

4. Fukuwatari T, Kawada T, Tsuruta M, Hiraoka T, Iwanaga T, Sugimoto E and Fushiki T (1997) Expression of the putative membrane fatty acid transporter (fat) in taste buds of the circumvallate papillae in rats. FEBS Lett 414, 461-464.

5. Galindo MM, Voigt N, Stein J, van Lengerich J, Raguse J-D, Hofmann T, Meyerhof W and Behrens M (2012) G proteincoupled receptors in human fat taste perception. Chem Senses 37, 123-139.

6. Geisler S and Zahm DS (2005) Afferents of the ventral tegmental area in the rat-anatomical substratum for integrative functions. J Comp Neurol 490, 270-294.

7. Gilbertson TA, Fontenot DT, Liu L, Zhang H and Monroe WT (1997) Fatty acid modulation of $\mathrm{k}^{+}$channels in taste receptor cells: gustatory cues for dietary fat. Am J Physiol 272, C1203-1210.

8. Godinot N, Yasumatsu K, Barcos ME, Pineau N, Ledda M, Viton F, Ninomiya Y, le Coutre J and Damak S (2013) Activation of tongue-expressed gpr40 and gpr120 by non caloric agonists is not sufficient to drive preference in mice. Neuroscience 250, 20-30.

9. Hajnal A, Smith GP and Norgren R (2004) Oral sucrose stimulation increases accumbens dopamine in the rat. Am $J$ Physiol Regul Integr Comp Physiol 286, R31-37.

10. Harden CJ, Jones AN, Maya-Jimenez T, Barker ME, Hepburn NJ, Garaiova I, Plummer SF and Corfe BM (2012) Effect of different long-chain fatty acids on cholecystokinin release in vitro and energy intake in free-living healthy males. $\mathrm{Br} J$ Nutr 108, 755-758.

11. Hirasawa A, Tsumaya K, Awaji T, Katsuma S, Adachi T, Yamada M, Sugimoto Y, Miyazaki S and Tsujimoto G (2005) Free fatty acids regulate gut incretin glucagon-like peptide-1 secretion through gpr120. Nat Med 11, 90-94.

12. Iino T, Sugimoto H, Watanabe A, Encinas JA, Liu N, Floeckner $\mathrm{J}$ and Bacon KB (2002) Molecular cloning and functional characterization of cynomolgus monkey (macaca fascicularis) cc chemokine receptor, ccr3. Cytokine 19, 276-286.

13. Imaizumi M, Takeda M and Fushiki T (2000) Effects of oil intake in the conditioned place preference test in mice. Brain Res 870, 150-156.

14. Iwakura H, Ariyasu H, Hosoda H, Yamada G, Hosoda K,
Nakao K, Kangawa K and Akamizu T (2011) Oxytocin and dopamine stimulate ghrelin secretion by the ghrelin-producing cell line, mgn3-1 in vitro. Endocrinology 152, 26192625.

15. Kawai $\mathrm{T}$ and Fushiki $\mathrm{T}$ (2003) Importance of lipolysis in oral cavity for orosensory detection of fat. Am J Physiol Regul Integr Comp Physiol 285, R447-454.

16. Laugerette F, Passilly-Degrace P, Patris B, Niot I, Febbraio M, Montmayeur JP and Besnard P (2005) Cd36 involvement in orosensory detection of dietary lipids, spontaneous fat preference, and digestive secretions. J Clin Invest 115, 3177-3184.

17. Liang N-C, Hajnal A and Norgren R (2006) Sham feeding corn oil increases accumbens dopamine in the rat. Am $J$ Physiol Regul Integr Comp Physiol 291, R1236-1239.

18. Matsumura S, Eguchi A, Mizushige T, Kitabayashi N, Tsuzuki $\mathrm{S}$, Inoue K and Fushiki T (2009) Colocalization of gpr120 with phospholipase-cbeta2 and alpha-gustducin in the taste bud cells in mice. Neurosci Lett 450, 186-190.

19. Meye FJ and Adan RAH (2014) Feelings about food: the ventral tegmental area in food reward and emotional eating. Trends Pharmacol Sci 35, 31-40.

20. Paxinos G and Franklin KBJ (2001) The Mouse Brain in Stereotaxic Coordinates, Second Edition, Academic Press, San Diego.

21. Pettit HO and Justice JJB (1991) Effect of dose on cocaine self-administration behavior and dopamine levels in the nucleus accumbens. Brain Res 539, 94-102.

22. Saitou K, Yoneda T, Mizushige T, Asano H, Okamura M, Matsumura S, Eguchi A, Manabe Y, Tsuzuki S, Inoue K and Fushiki T (2009) Contribution of gustation to the palatability of linoleic acid. Physiol Behav 96, 142-148.

23. Takeda M, Imaizumi M and Fushiki T (2000) Preference for vegetable oils in the two-bottle choice test in mice. Life Sci 67, 197-204.

24. Tucker RM, Mattes RD and Running CA (2014) Mechanisms and effects of "fat taste" in humans. Biofactors 40, 313-326

25. Willuhn I, Wanat MJ, Clark JJ and Phillips PEM (2010) Dopamine signaling in the nucleus accumbens of animals selfadministering drugs of abuse. Curr Top Behav Neurosci 3, 29-71.

26. Wise RA and Rompre PP (1989) Brain dopamine and reward. Annu Rev Psychol 40, 191-225.

27. Wise RA (2006) Role of brain dopamine in food reward and reinforcement. Philos Trans R Soc Lond B Biol Sci 361, $1149-1158$.

28. Yoneda T, Saitou K, Asano H, Mizushige T, Matsumura S, Eguchi A, Manabe Y, Tsuzuki S, Inoue K and Fushiki T (2009) Assessing palatability of long-chain fatty acids from the licking behavior of balb/c mice. Physiol Behav 96, 735741.

29. Yoneda T, Saitou K, Mizushige T, Matsumura S, Manabe Y, Tsuzuki S, Inoue K and Fushiki T (2007) The palatability of corn oil and linoleic acid to mice as measured by short-term two-bottle choice and licking tests. Physiol Behav 91, 304309.

30. Yoneda T, Taka Y, Okamura M, Mizushige T, Matsumura S, Manabe Y, Tsuzuki S, Inoue K and Fushiki T (2007) Reinforcing effect for corn oil stimulus was concentration dependent in an operant task in mice. Life Sci 81, 1585-1592. 